Chirurg 2014 $\cdot 85: 1110$

DOI 10.1007/s00104-014-2908-6

Online publiziert: 23. November 2014

c) Springer-Verlag Berlin Heidelberg 2014

K. Lorenz · J. Hein · H. Dralle

Universitätsklinik für Allgemein-, Viszeral- und Gefäßchirurgie, Medizinische Fakultät,

Universitätsklinikum Halle, Halle (Saale)

\title{
Primärer Hyperparathyreoidismus nach Adipositaschirurgie
}

\section{Ergebnisse}

Die präoperative Bildgebung ergab folgende richtig- vs. falsch-positive Ergebnisse: Sonographie 6 vs. 2; MIBI-Szintigraphie 5 vs. 1; 4 -D-Computertomographie 1 vs. 0. Acht Patienten waren symptomatisch. Bei 7 Patienten mit fokussiertem Zugang wurde zweimal konvertiert, 3 Patienten primär bilateral exploriert. Histologisch ergaben sich 7 singuläre Adenome, 1 Doppeladenom und 2 Mehrdrüsenerkrankungen (MDE). IOPTH dokumentierte bei 7 Einzeladenomen die erfolgreiche Parathyreoidektomie. Im Verlauf zeigten 5 Patienten PTH und Serumkalzium im Normbereich (Mittel: Serumkalzium 9,3 mg/dl; PTH 73 pg/ml; 25-Vitamin-D $44 \mathrm{ng} / \mathrm{ml}$ ), 5 Patienten (3 mit Einzeladenom, 2 mit MDE) zeigten bei Normokalzämie ein persistierend erhöhtes PTH. Ein pHPT-Rezidiv mit MDE wurde nach 46 Monaten reoperiert.

\section{Diskussion und Fazit}

Retrospektiv zeigten in 2 Jahren 10 weibliche Patienten (mittleres Alter 58,4 Jahre) im Mittel 67 Monate nach RYGBP einen pHPT bei durchschnittlicher Body-MassIndex-Abnahme von $16,5 \mathrm{~kg} / \mathrm{m}^{2}$ und $59,7 \%$ Gewichtsverlust. Präoperativ betrugen im Mittel Serumkalzium 10,8 mg/ dl (8,5-10,5), Parathormon (PTH) 155 pg/ $\mathrm{ml}$ (15-65), 25-Vitamin-D $32 \mathrm{ng} / \mathrm{ml}$ (20$80)$. Operationsindikation waren die Biochemie, skelettale und muskuläre Symptome. IOPTH (intraoperative Parathormonschnellbestimmung) -Erfolgskriterium war 50\% Abfall vom Basalwert, die Nachsorge betrug im Mittel 19 Monate.
Die sehr kleine Serie erlaubt zwar keine soliden Schlussfolgerungen, sie zeigt aber die Problematik bezüglich der Diagnostik, Differenzierung und adäquaten Behandlung des gestörten Kalziummetabolismus nach Adipositaschirurgie sehr deutlich auf. Zum pHPT nach RYGBP wird richtigerweise aufgrund der ungeklärten Fragen auf Folgendes hingewiesen:

1. Die Diagnose eines pHPT nach RYGBP erfordert obligat das Vorliegen einer Hyperkalzämie. Ein bei Normokalzämie allein erhöhtes Parathormon bedeutet die typische Konstellation eines SHPT nach RYGBP und beinhaltet damit keine Operationsindi- kation. Sowohl diagnostisch als auch therapeutisch sollte ein normaler Vitamin-D-Spiegel vorliegen, um präund postoperativ die zusätzliche Konstellation eines SHPT bestmöglich auszuschließen.

2. Ob die Inzidenz einer MDE beim pHPT nach RYGBP erhöht ist, kann durch die vorliegende Untersuchung nicht belegt, aber auch nicht ausgeschlossen werden. Der intraoperative Ausschluss einer MDE erfordert daher auch bei vermeintlichem Einzeladenom den Einsatz strenger IOPTH-Kriterien (Abfall des IOPTH 15 min nach Parathyreoidektomie unterhalb $50 \%$ des oberen PTH-Normbereichs) und gleichzeitig im Zweifelsfall eine bilaterale Nebenschilddrüsenexploration.

\section{Korrespondenzadresse}

Prof. Dr. K. Lorenz

Universitätsklinik für Allgemein-, Viszeralund Gefäßchirurgie, Medizinische Fakultät, Universitätsklinikum Halle,

Ernst-Grube-Str. 40, 06097 Halle (Saale) kerstin.lorenz@uk-halle.de

Interessenkonflikt. K. Lorenz, J. Hein und H. Dralle geben an, dass kein Interessenkonflikt besteht.

\section{Literatur}

1. Jin J, Robinson AV, Hallowell PT et al (2007) Increases in parathyroid hormone after gastric bypass surgery appear to be of secondary nature. Surgery 142:914-920

2. Campos GM, Ziemelis M, Paparodis R et al (2014) Laparoscopic reversal of Roux-en Y gastric bypass: technique and utility for treatment of endocrine complications. Surg Obes Relat Dis 10:36-43

3. Jeon JH, Cheung DY, Lee SJ et al (2014) Endoscopic resection yields reliable outcomes for small rectal neuroendocrine tumors. Dig Endosc. doi:10.1111/ den.12232 